\title{
Wir laden Sie herzlich nach Aachen ein!
}

E $s$ ist uns eine besondere reude, Sie zum ersten Kongress der drei großen allergologischen Fachgesellschaften im September 2004 in die Kaiserstadt Aachen im Dreiländereck von Deutschland, Belgien und den Niederlanden einzuladen.

Wir hoffen, dass Sie vom Charme der Stadt und auch ihrer Umgebung sowie von ihren vielfältigen Kontrasten überrascht sein werden, die sich aus der besonderen historischen Tradition zwischen Krönungsort der meisten deutschen Kaiser und dem Standort einer der führenden Technischen Hochschulen Europas ergeben.

Wir tagen im Eurogress, dem zentralen Kongressort in Aachen, nur wenige Gehminuten entfernt vom berühmten historischen Zentrum mit dem Dom Karls des Großen und dem heutigen Rathaus, an dessen Stelle früher die Kaiserpfalz des großen Karolingers stand. Unser Begleitprogramm wird Ihnen in der Stadt einige besonders interessante Orte vorstellen. So werden ein

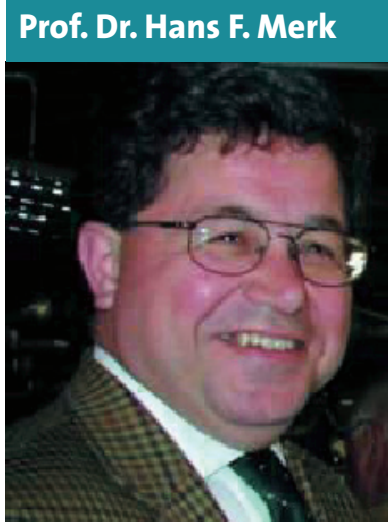

Klinik für Dermatologie und Allergologie, Universitätsklinikum der RWTH, Aachen, Kongresspräsident Allergie-Kongress 2004

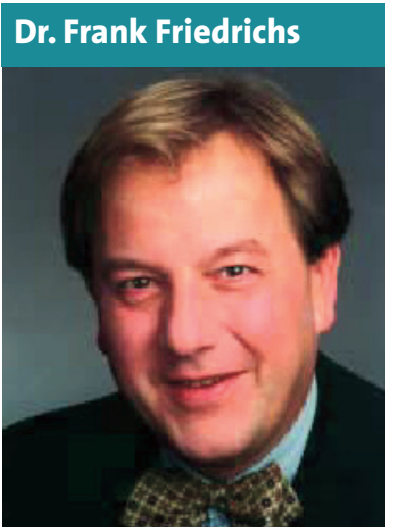

Kinderärztliche Praxisgemeinschaft AachenLaurensberg, Aachen, Kongresspräsident Allergie-Kongress 2004
Orgelkonzert im Dom und der Gesellschaftsabend in der Aula Carolina stattfinden.

Das wissenschaftliche Programm spiegelt die Vielfalt der Themen in der modernen Allergologie wider, aber auch die unterschiedlichen Rahmen, in denen sie vermittelt werden können und wie sie sich in den vergangenen Jahren in teils unterschiedlicher Gewichtung in den drei Fachgesellschaften etabliert haben. Der gegenwärtige Einfluss gerade
Aachener Politiker auf die Gestaltung der Gesundheitspolitik wie auch die eine Woche nach dem Kongress stattfindende, mit Spannung erwartete Kommunalwahl in Nordrhein-Westfalen legen nahe, auch unser politisches Umfeld auf diesem Kongress zu diskutieren. Am Sonntag öffnen wir die Türen im Rahmen einer pina-Veranstaltung weit für alle an der Allergie Interessierten. Die Programmvorschau können Sie sowohl dem Vorprogramm, das einer Teilauflage dieser Ausgabe des Allergo Journal beiliegt, als auch in jeweils aktualisierter Form der Homepage des Kongresses (www.allergie-kongress-2004.de) entnehmen.

Wir hoffen, dass Ihnen dieses Programm und der Ausblick auf den Kongress zusagen und wir Sie im September 2004 in der Kaiserstadt begrüßen dürfen.

Prof. Dr. Hans F. Merk, Aachen; Dr. Frank Friedrichs, Aachen

\section{Allergie-Kongress Aachen 2004 auf einen Blick}

\section{Veranstalter \\ Ärzteverband Deutscher Allergologen (ÄDA) e. V.

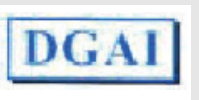 \\ Deutsche Gesellschaft für Allergologie und klinische Immunologie (DGAI) e. V.

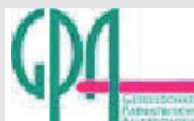 \\ Gesellschaft für Pädiatrische Allergologie und Umweltmedizin (GPA) e. V.}

Tagungspräsidenten Univ.-Prof. Dr. med. Hans F. Merk Dr. med. Frank Friedrichs

\section{Kongressorganisation}

Service Systems GbR

Carin Fresle und Ursula Raab

Blumenstraße 14, 63303 Dreieich

Tel.: (oo 49-61 03) 63657

Fax: (0о 49-61 03) 67674

E-Mail: aeda@sersys.de

\section{Kongressbüro Aachen}

Kinderärztliche Praxisgemeinschaft

Aachen-Laurensberg

Elke Dovern

Rathausstraße 10, 52072 Aachen

Tel.: (oo 49-2 41) 171096

Fax: (oo 49-2 41) 174349

E-Mail: dovern@kinderarztpraxis-

laurensberg.de

\section{Vorprogramm}

Das offizielle Vorprogramm des Allergie-Kongresses 2004 kann bei der Kongressorganisation kostenlos angefordert werden.

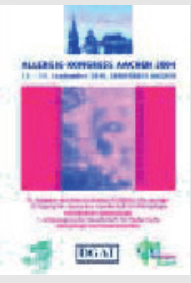

Internet

www.allergie-kongress-2004.de

Anmeldeschluss für Abstracts

Wissenschaftliche Arbeiten, die in Aachen als Vorträge oder Poster präsentiert werden sollen, können bis zum 16. Mai 2004 eingereicht werden. 